\title{
Upper airway factors that protect against obstructive sleep apnoea in healthy older males
}

\author{
Thomas Carlisle ${ }^{1,2,4}$, Elliott R. Carthy ${ }^{1,2,4}$, Martin Glasser ${ }^{1,2}$, Peter Drivas $^{3}$, \\ Alison McMillan ${ }^{1,2}$, Martin R. Cowie ${ }^{3}$, Anita K. Simonds ${ }^{1,2}$ and Mary J. Morrell ${ }^{1,2}$ \\ Affiliations: ${ }^{1}$ Academic Unit of Sleep and Breathing, National Heart and Lung Institute, Imperial College, \\ London, UK. ${ }^{2}$ National Institute for Health Research (NIHR) Respiratory Biomedical Research Unit, Royal \\ Brompton and Harefield Foundation Trust, London, UK. ${ }^{3} \mathrm{NIHR}$ Cardiovascular Biomedical Research Unit, Royal \\ Brompton and Harefield Foundation Trust, London, UK. ${ }^{4}$ Both authors contributed equally.
}

Correspondence: Mary J. Morrell, Academic Unit of Sleep and Ventilation, Royal Brompton Hospital, Sydney Street, London, SW3 6NP, UK. E-mail: m.morrellaimperial.ac.uk

ABSTRACT The prevalence of obstructive sleep apnoea (OSA) increases with age, yet the risk factors for OSA in older people remain poorly understood.

This study aimed to define the age-related changes in upper airway morphology in carefully matched groups of healthy older $(>60$ years, $n=11)$ and younger $(<40$ years, $n=14)$ males, using direct (magnetic resonance imaging (MRI)) and indirect (acoustic reflection) imaging.

The median (interquartile range) combined retropalatal and retroglossal pharyngeal length was greater in older than in younger males (older $8.8(7.8-9.0) \mathrm{cm}$, younger $7.8(7.0-8.3) \mathrm{cm}$; $\mathrm{p}=0.03$ ), as was the soft palate cross-sectional area (older $43.1(36.0-48.8) \mathrm{cm}^{2}$, younger $35.3(30.5-40.5) \mathrm{cm}^{2} ; \mathrm{p}=0.03$ ), parapharyngeal fat pad diameter (older $1.7(1.4-2.2) \mathrm{cm}$, younger $1.2(1.0-1.8) \mathrm{cm} ; \mathrm{p}=0.03)$ and crosssectional area of the fat pads (older $13.8(9.1-17.1) \mathrm{cm}^{2}$; younger $7.4(5.9-13.0) \mathrm{cm}^{2} ; \mathrm{p}=0.02$ ) as measured by MRI. Using acoustic reflection, pharyngeal calibre (older $4.8(3.8-6.6) \mathrm{cm}^{2}$, younger $3.4(2.8-4.6) \mathrm{cm}^{2}$; $\mathrm{p}=0.03$ ), pharyngeal volume (older $35.1(30.9-55.4) \mathrm{cm}^{3}$, younger $\left.27.2(22.7-44.2) \mathrm{cm}^{3} ; \mathrm{p}=0.04\right)$ and glottis area (older $2.7(2.1-3.9) \mathrm{cm}^{2}$, younger $\left.1.3(1.1-1.9) \mathrm{cm}^{2} ; \mathrm{p}=0.003\right)$ were also larger in older participants compared with younger participants. There was no difference in craniofacial measures between groups, including volumetric data and hyoid bone position.

The larger pharyngeal calibre observed in older males may be compensating for an age-related enlargement in pharyngeal soft tissue that predisposes to OSA.

@ERSpublications

Pharyngeal factors predisposing to sleep apnoea increase with age; a larger calibre protected against airway collapse http://ow.ly/v746x Brompton and Harefield NHS Foundation Trust.

Conflict of interest: Disclosures can be found alongside the online version of this article at erj.ersjournals.com 


\section{Introduction}

The prevalence of obstructive sleep apnoea (OSA) is increasing, with current estimates for moderate-tosevere OSA (apnoea-hypopnoea index (AHI) $\geqslant 5$ events $\cdot \mathrm{h}^{-1}$ ) in $30-70$-year-olds being $34 \%$ of males and $17 \%$ of females [1]. Notably, this is a large increase compared with earlier reports of $24 \%$ and $9 \%$, respectively [2], which is likely to be associated with secular trends in the established risk factors of obesity and demographic ageing. The current estimate of OSA prevalence in older males ( $\geqslant 60$ years) is $30-50 \%$ [2], with evidence that disease severity also increases over time [3]. However, the clinical diagnosis of OSA in older people is problematic due to differences in the expression of symptoms such as excessive daytime sleepiness [4]. Improved phenotyping of OSA in older people will enable more efficient diagnosis and initiation of effective therapy.

The pharyngeal morphological risk factors for OSA are well established in the general population [5-8]; however, the data regarding age-related differences are unclear. Pharyngeal lengthening may be associated with greater OSA severity [9] and there is also evidence that pharyngeal lengthening occurs with increasing age [10]. Surrogate markers of pharyngeal lengthening include increased facial length [11] and a more caudally located hyoid bone [12]. Soft tissue size is also associated with increased risk of OSA, including tongue, soft palate and fat pad volumes causing a reduction in pharyngeal calibre in middle-aged participants [8, 13]. Malhotra et al. [10] used a small cohort of males and females to show that parapharyngeal fat pad volume correlated with age, thereby suggesting that soft tissue size is also a risk factor for OSA in older people. For pharyngeal calibre some [14-16], but not all studies [17-20] have shown that calibre is increased in older compared with younger people. These inconsistencies may be due to undiagnosed OSA in the older participants studied.

The aims of this study were to investigate the effects of age per se on upper airway morphology in healthy older and younger males, using noninvasive direct and indirect imaging techniques (magnetic resonance imaging (MRI) and acoustic reflection, respectively). We tested the hypotheses that pharyngeal calibre is decreased, and pharyngeal length and soft tissue size are increased in older healthy males, when compared with younger healthy males, which may predispose to pharyngeal collapse.

\section{Methods}

14 healthy younger males (20-40 years old) and 11 healthy older males ( $>60$ years old), that had been carefully group-matched for body mass index (BMI), neck circumference, subjective daytime sleepiness, AHI and oxygen desaturation index (ODI) were studied. Participants were screened using respiratory polygraphy to exclude OSA. Participants had no known cardiorespiratory disease, no previous upper airway surgery and no sleep disorder. Those who had an AHI $<5$ events $\cdot \mathrm{h}^{-1}$ and met all the other inclusion/ exclusion criteria were invited to undergo direct MRI of the upper airway and indirect imaging using acoustic reflection. Acoustic reflection was chosen as the most appropriate modality for measuring pharyngeal length and calibre, and MRI as a measure of soft tissue anatomy surrounding the airway. The sample size was based on previously published work that had yielded significant results [21]. Informed written consent was obtained from each participant and the study was ethically approved (London-Chelsea ethics committee REC number 09/H0708/24).

\section{Respiratory polygraphy}

Respiratory polygraphy (SOMNOscreen; Somnomedics GmBH, Randersacker, Germany) was carried out either in the participants home or at the sleep laboratory, according to the participants' preference. Heart rate (via ECG), snoring, breathing effort (respiratory inductance plethysmography), airflow at the nostrils (cannula with a pressure transducer) and arterial oxygen saturation were measured. Participants were asked to complete a sleep diary documenting time in bed (from lights off to awakening) and any periods of extended wakefulness. AHI $\geqslant 5$ events $\cdot \mathrm{h}^{-1}$ was used to define the presence of OSA. Data were analysed using the American Academy of Sleep Medicine recommended scoring criteria [22]. Hypopnoeas were defined as $\mathrm{a} \geqslant 30 \%$ reduction in airflow accompanied by $\mathrm{a} \geqslant 4 \%$ desaturation lasting for $\geqslant 10 \mathrm{~s}$.

\section{Direct pharyngeal imaging: MRI}

Two-dimensional MRI scans were obtained using a 1.5-T magnetic resonance scanner (Siemens Avanto; Siemens Healthcare, Erlangen, Germany) (T1 weighted, 2D axial and sagittal spin echo sequences). Participants were asked to lie supine with their head secured in a neutral anatomical position (Frankfort plane). Breathing was via the same mouthpiece as for the acoustic reflection measurements to ensure the pharyngeal anatomy was similarly positioned for both analyses. Sequential images were obtained from the top of the cranium to the superior border of the trachea. Sagittal (3-mm slice thickness) and axial (5-mm slice thickness) views were analysed using ImageJ software (version 1.46r; US National Institutes of Health, Bethesda, MD, USA). Consistent with previous studies [10, 21], sagittal anatomy (fig. 1a) was analysed 

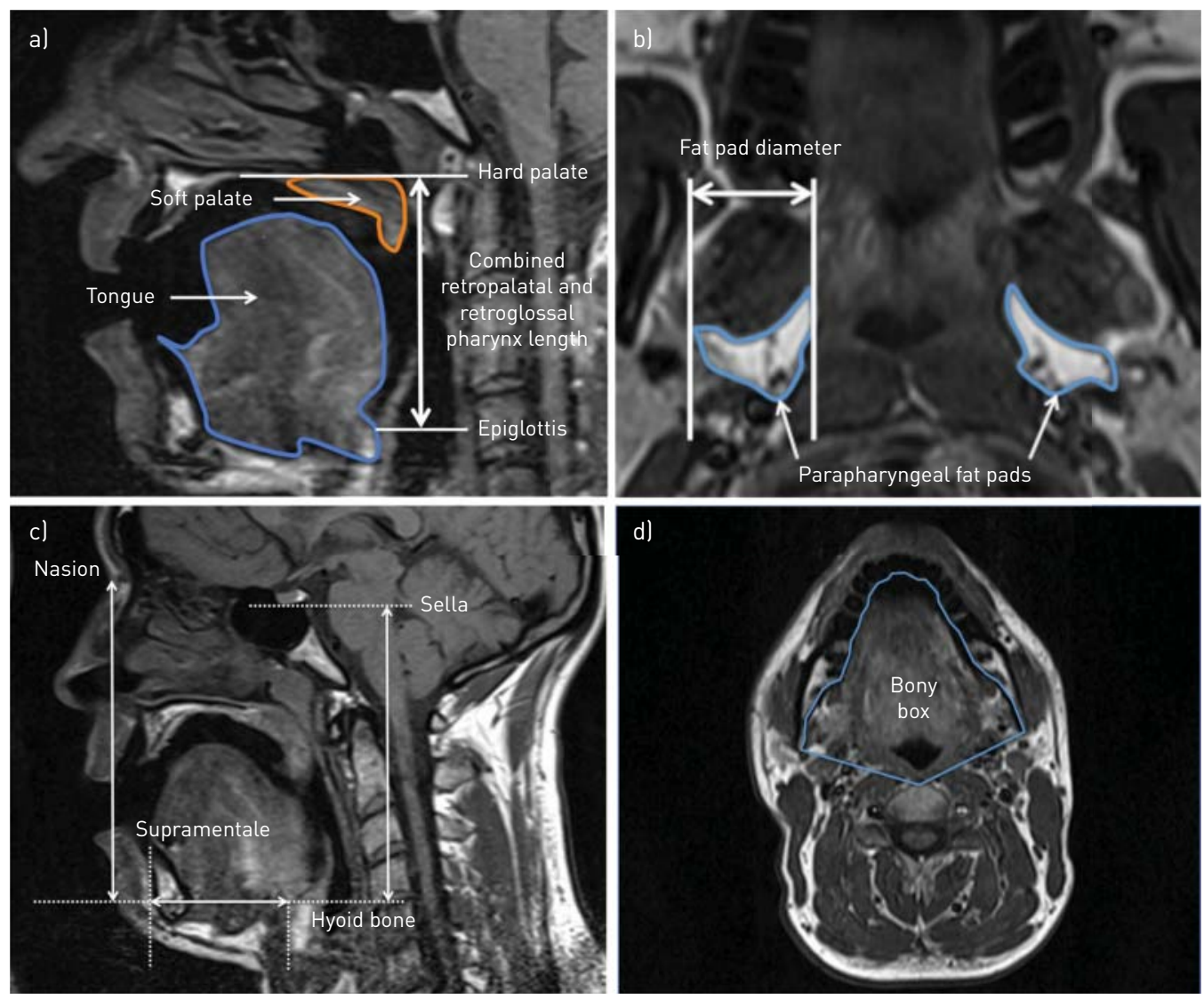

FIGURE 1 T1-weighted magnetic resonance imaging sagittal slices denoting a) the combined retropalatal and retroglossal pharyngeal length and soft tissue measures and c) hyoid bone location, and axial slices denoting b) the fat pad measures and d) the bony box measures used for this study.

using the midsagittal slice, chosen by eye as the slice showing maximum pharyngeal calibre. The combined retropalatal and retroglossal pharyngeal length was measured from the hard palate to the base of the epiglottis, and further subdivided into retropalatal and retroglossal lengths. The retropalatal region was measured from the hard palate to the tip of the soft palate, and the retroglossal region measured from the tip of the soft palate to the glottis. The soft palate cross-sectional area and tongue cross-sectional area were measured using the same sagittal slice. Axial slices (fig. 1b) were chosen by eye at the level of the inferior border of the maxilla, with anatomical parameters of interest comprising the parapharyngeal fat pad diameter and cross-sectional area.

Further MRI parameters included midsagittal cephalometric measures of the vertical distance from nasion to hyoid, sella to hyoid and anterior-posterior distance from supramentale to hyoid using established methods (fig. 1c) [23]. Volumetric measures were made of craniofacial structures from axial MRI slices including the bony box volume, tongue volume and the ratio of the two. The bony box was measured using methods adapted from those of SCHWAB et al. [8]. Sequential cross-sectional areas of the bony box region and tongue measured on each slice form the top of the mandible moving inferiorly (fig. 1d), the means of which were used to calculate the volume (mean cross-sectional area $\times$ number of slices $\times$ slice thickness). A single investigator performed all MRI analyses, blinded to the study group.

\section{Indirect pharyngeal imaging: acoustic reflection}

Acoustic reflection was used as a noninvasive technique for measuring internal dimensions of the pharynx (A1 Executive Acoustic Rhinometer; GM Instruments Ltd, Kilwinning, UK). The advantage of this technique was that it could be used at the bedside $[17,24]$. Participants were asked to lie supine with their head supported in the Frankfort plane using one pillow. The acoustic reflection device was positioned perpendicular to the floor, and participants were asked to form a seal with their lips over the mouthpiece attached to the acoustic wave tube and sound pulse generator. Participants were instructed to breathe through their mouth, with minimal movement of the head, neck and jaw. After 5 min of supine rest, at least 50 traces were recorded at end-tidal expiration, a point in the respiratory cycle that has been previously 
validated for pharyngeal measurements $[17,18]$. Acoustic reflection was measured in all participants by a single investigator and analysed offline using a computer algorithm that minimised human bias.

Serial measurements of cross-sectional area were made at intervals of $0.2 \mathrm{~mm}$ along part of the oral cavity starting from the end of the mouthpiece, and the retroglossal pharynx in order to construct a trace of crosssectional area plotted as a function of distance [17, 24]. From this we derived measurements of oral/ retroglossal pharyngeal length, mean pharyngeal area, glottis cross-sectional area and pharyngeal volume (mean pharyngeal area $\times$ by oral/retroglossal pharyngeal length) (fig. 2). For the purposes of acoustic reflection measurements, the pharynx is conventionally defined as the region between the end of the mouthpiece at the level of the incisors to the glottis; the latter is defined as the smallest cross-sectional area distal to the largest cross-sectional area on the acoustic reflection trace (fig. 2). Measurements were calculated for each individual trace and averaged to obtain mean values per participant. Prior to the study, the reproducibility of the acoustic reflection technique was assessed. A threshold coefficient of variation of $10 \%$ was taken as an acceptable within-test variability for these measurements [25]. Acoustic reflection measurements of pharyngeal cross-sectional area (seated and supine) in 17 healthy volunteers on two separate occasions at least $24 \mathrm{~h}$ apart and at the same time of day $( \pm 2 \mathrm{~h})$ showed excellent reproducibility with a coefficient of variation $<10 \%$ in 11 out of the 17 subjects.

\section{Statistics}

After formally testing the data for normality, comparisons between age groups were performed using Mann-Whitney U-tests. Data are presented as median (interquartile range). Statistical significance was determined using a threshold p-value of 0.05. Analyses were performed using SPSS (IBM Statistics 19; IBM Corporation, Armonk, NY, USA).

\section{Results}

Table 1 shows the participant demographics of the healthy younger $(n=14)$ and older $(n=11)$ male groups. By design, there was a significant difference in the median age between groups. The groups were well matched for all other characteristics. Table 2 shows the sleep parameters for both groups. The AHI and ODI were similar between groups. All participants underwent measures of upper airway MRI and acoustic reflection.

\section{Direct pharyngeal imaging: MRI}

Combined retropalatal and retroglossal pharyngeal length

The combined retropalatal and retroglossal pharyngeal length was significantly greater in older than in younger males (older $8.8(7.8-9.0) \mathrm{cm}$, younger $7.8(7.0-8.3) \mathrm{cm} ; \mathrm{p}=0.03)$ (fig. 3a). When the combined

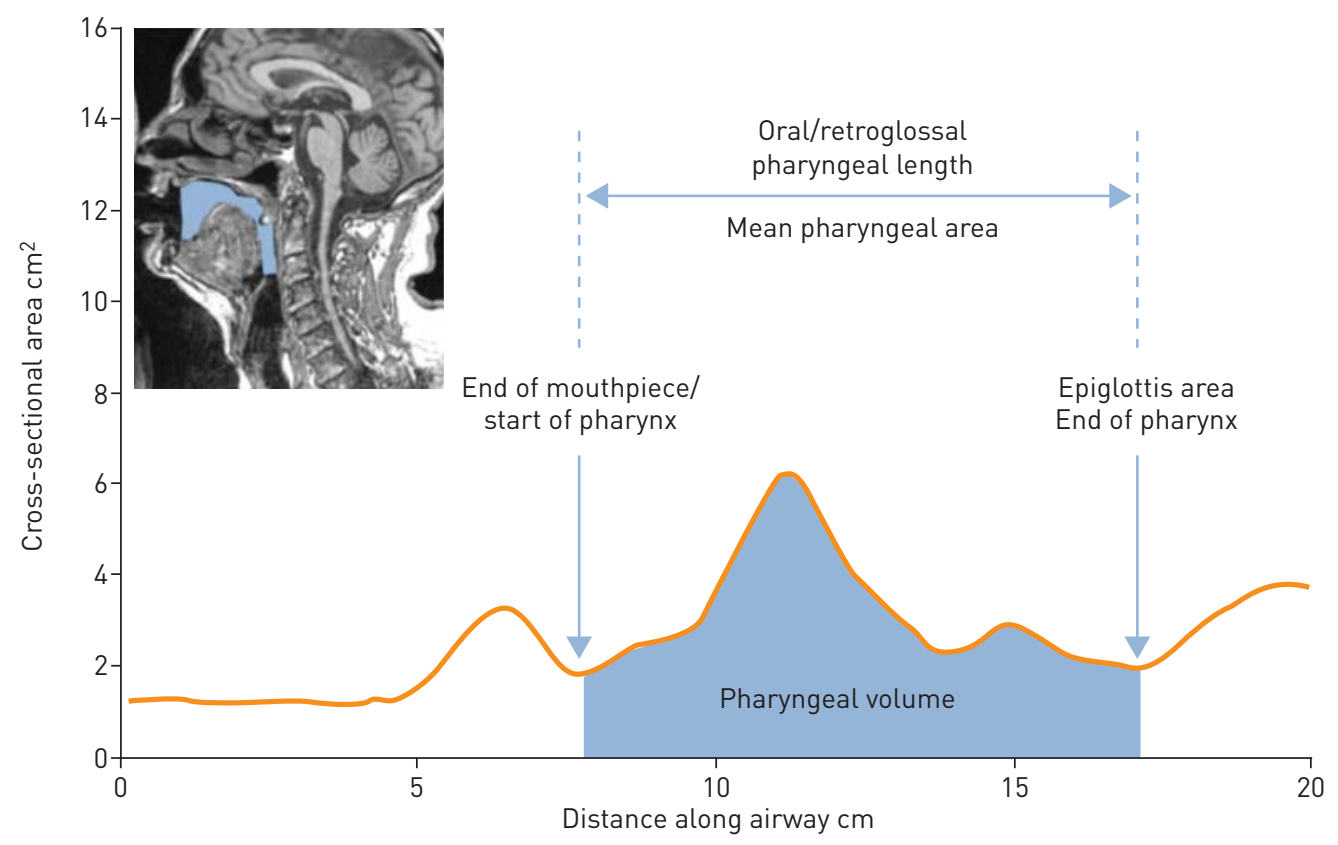

FIGURE 2 Acoustic reflection trace of the upper airway. The pharynx was designated as the region between the end of the mouthpiece and the glottis (highlighted on inset). Mean pharyngeal area was obtained by calculating the mean of serial measurements along the length of the pharynx. Pharyngeal volume was obtained as a product of oral/retroglossal pharyngeal length and mean pharyngeal area. 
Younger males

Subjects $\mathrm{n}$
Age years
Height $\mathrm{m}$
Body mass index $\mathrm{kg} \cdot \mathrm{m}^{-2}$
Neck circumference $\mathrm{cm}$
Epworth sleepiness score

Older males

11

$65(63-69)$

$1.8(1.7-1.9)$

$27(25-29)$

$40(39-40)$

$5(4-6)$

Data are presented as median (interquartile range), unless otherwise stated. There were no significant differences $\left(p=0.05\right.$ ) between groups for any parameter (except for age). ${ }^{\#}$ : out of a total possible score of 24.

retropalatal and retroglossal pharynx was subdivided, the retropalatal length was significantly greater in older males (older $3.6(3.4-4.0) \mathrm{cm}$, younger $3.0(2.7-3.3) \mathrm{cm} ; \mathrm{p}=0.003)$ (fig. 3b), with no difference in the retroglossal length (older $4.6(4.2-5.1) \mathrm{cm}$, younger $4.5(4.2-5.3) \mathrm{cm} ; \mathrm{p}>0.99)$ (fig. 3c).

\section{Soft tissues}

Older males had a bigger soft palate cross-sectional area (older $43.1(36.0-48.8) \mathrm{cm}^{2}$, younger 35.3 (30.5-40.5) $\mathrm{cm}^{2} ; \mathrm{p}=0.03$ ) (fig. 4a), parapharyngeal fat pad diameter (older $1.7(1.4-2.2) \mathrm{cm}$, younger 1.2 $(1.0-1.8) \mathrm{cm} ; \mathrm{p}=0.03$ ) (fig. $4 \mathrm{~b}$ ) and cross-sectional area (older $13.8(9.1-17.1) \mathrm{cm}^{2}$, younger $7.4(5.9-13.0) \mathrm{cm}^{2}$; $\mathrm{p}=0.02$ ) (fig. $4 \mathrm{c}$ ) than younger males. There was no difference in tongue size between groups (older 294.2 (289.8-324.9) $\mathrm{cm}^{2}$, younger 322.9 (309.1-337.1) $\mathrm{cm}^{2} ; \mathrm{p}=0.07$ ) (fig. 4d).

\section{Craniofacial structures}

There was no significant difference in the craniocaudal (nasion and sella to hyoid distance) or anteroposterior (supramentale to hyoid distance) location of the hyoid bone between younger and older participants (table 3). There was also no difference in the bony box or tongue volumes between groups (table 3).

\section{Indirect pharyngeal imaging: acoustic reflection \\ Oral/retroglossal pharyngeal length}

In contrast to the combined retropalatal and retroglossal pharyngeal length measured using MRI, oral/ retroglossal pharyngeal length measured using acoustic reflection was similar between younger and older males (older $8.8(7.6-9.6) \mathrm{cm}$, younger $8.1(7.6-8.7) \mathrm{cm}$; $\mathrm{p}=0.6)$ (fig. 5a).

\section{Pharyngeal calibre}

Pharyngeal calibre was significantly greater in older males than in younger males using the mean pharyngeal area (older $4.8(3.8-6.6) \mathrm{cm}^{2}$, younger $\left.3.4(2.8-4.6) \mathrm{cm}^{2} ; \mathrm{p}=0.03\right)$ (fig. 5b), glottis cross-sectional area (older $2.7(2.1-3.9) \mathrm{cm}^{2}$, younger $1.3(1.1-1.9) \mathrm{cm}^{2} ; \mathrm{p}=0.003$ ) (fig. $5 \mathrm{c}$ ) and pharyngeal volume (older 35.1 (30.9-55.4) $\mathrm{cm}^{3}$, younger $\left.27.2(22.7-44.2) \mathrm{cm}^{3} ; \mathrm{p}=0.04\right)$ (fig. $5 \mathrm{~d}$ ).

\section{Discussion}

The main findings of this study were that older males had greater pharyngeal calibre measured using acoustic reflection, and a longer combined retropalatal and retroglossal pharyngeal length, and larger soft palate and parapharyngeal fat pad size measured using MRI than younger males. These age-related pharyngeal morphological changes were independent of BMI and neck circumference.

The impact of age on pharyngeal calibre has been debated, with some [14-16] but not all [10, 17-20] studies finding greater pharyngeal calibre in older compared with younger males. This equivocal evidence may be due to poor screening and exclusion of undiagnosed OSA, as the prevalence of OSA is known to increase with age $[1,2]$. In the present study, appropriate screening ensured the exclusion of undiagnosed OSA, with groups well matched for BMI, neck circumference, subjective daytime sleepiness, AHI, ODI and craniofacial morphology. This approach created a model to establish the effect of age per se on pharyngeal calibre.

To our knowledge, there is only one additional study investigating the effects of age on pharyngeal soft tissue size [10]. This study found that older age correlated with increased soft palate and pharyngeal length in females but not males, and parapharyngeal fat pad thickness in males and females. No significant difference was found in pharyngeal volume. In the present study, an association between increasing age and 
TABLE 2 Sleep parameters as measured using respiratory polygraphy

Younger males

\section{Subjects $\mathrm{n}$}

Time in bed min

AHI events $\cdot h^{-1}$

Supine AHI events $\cdot h^{-1}$

$\mathrm{ODI}^{\#}$ events $\cdot \mathrm{h}^{-1}$

Time in bed spent supine \%

Time in bed spent lateral \%
14

$383(380-509)$

$0.8(0.6-1.4)$

$1.2(0.9-2.0)$

$0.8(0.3-1.9)$

$42(19-71)$

$57(29-75)$
Older males

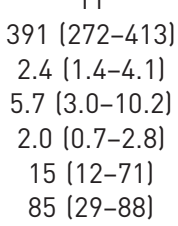

Data are presented as median (interquartile range), unless otherwise stated. There were no significant differences $(p=0.05)$ between groups for any parameter. AHI: apnoea-hypopnoea index; ODI: oxygen desaturation index. ${ }^{\#}$ : events were defined as desaturations of $\geqslant 4 \%$.

greater soft palate and parapharyngeal fat pad cross-sectional area was found, independent of BMI and neck circumference and, therefore, not related to differences in body fat. However, larger soft tissue volume has been previously correlated with smaller pharyngeal calibre [8], conflicting with the finding in the present study that larger pharyngeal calibre was observed alongside greater soft palate volume. We speculate that the large pharyngeal calibre in our participants was protective against OSA; thus, by screening out OSA, we probably preselected older males with larger pharyngeal calibre.

Functional compensation for the identified morphological risk factors within this healthy older male group may also have occurred, thereby protecting them from OSA. Patients with OSA have greater genioglossus activity at rest than healthy male controls, reflecting a neuromuscular response to a more collapsible pharynx [26, 27]. Moreover, genioglossus muscle activity is also raised in healthy middle-aged males compared with younger males $[28,29]$, suggesting that the reduction in muscle activity after sleep onset may predispose to OSA. Clinically, improved pathophysiological knowledge of OSA in older people would enable identification of those needing therapeutic intervention to prevent the cardiovascular and neurocognitive sequelae associated with OSA.

A strength of the present study is that two measurement techniques were used to measure the upper airway. Combined retropalatal and retroglossal pharyngeal length measured using MRI was found to be greater in older than in younger healthy males, but oral/retroglossal pharyngeal length measured using acoustic reflection was similar between groups. This finding of increased length with age is consistent with previous work [10] and an increased risk of OSA [9]. The difference between methodologies is probably explained by the anatomical regions measured. The MRI measure of the combined retropalatal and retroglossal pharyngeal length was taken vertically from the hard palate to the base of the glottis, whereas the acoustic reflection measurement of oral/retroglossal pharyngeal length included the oral cavity but less of the retropalatal region due to the soft palate abutting against the posterior pharyngeal wall, sealing off the nasopharynx. The retropalatal, but not retroglossal, length was significantly greater in older healthy males, which is consistent with studies demonstrating that the retropalatal region of the pharynx is a common site of collapse [30].

The difference in combined retropalatal and retroglossal pharyngeal length was found to be independent of craniofacial morphology. Inferior and posterior displacement of the hyoid bone has been associated with OSA,
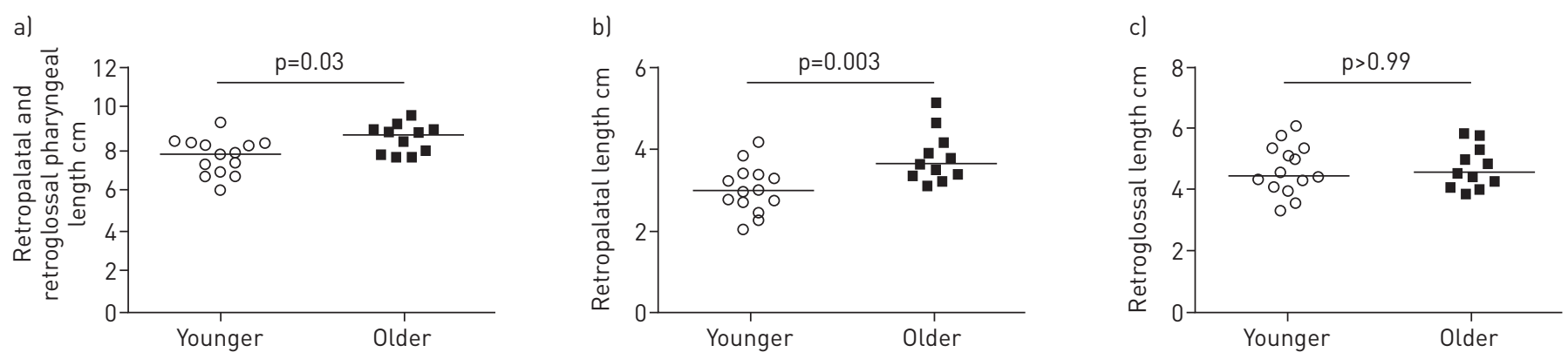

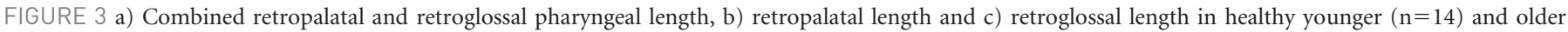
$(n=11)$ male participants as measured from magnetic resonance images. Median data are denoted by horizontal lines on each plot. 

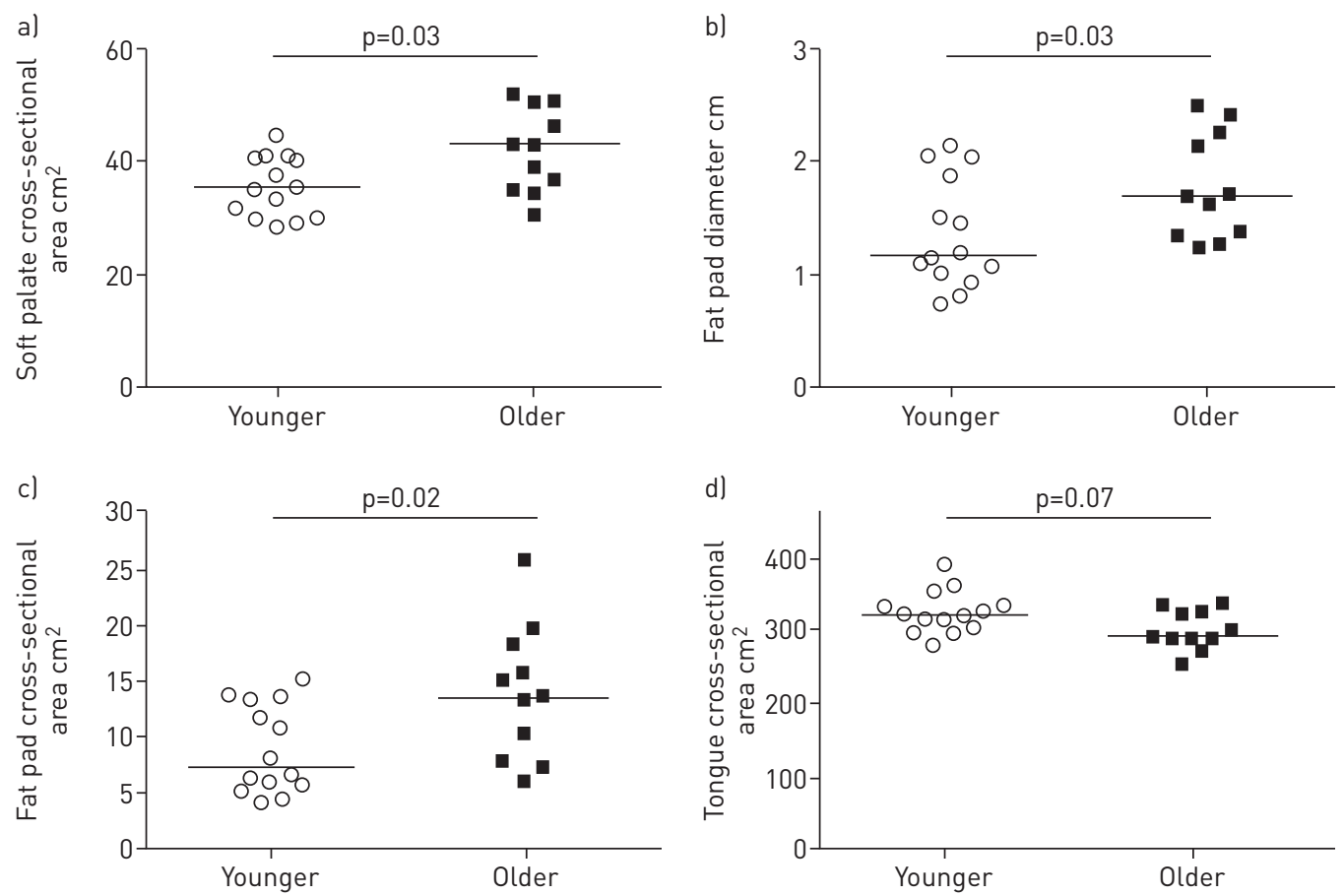

FIGURE 4 Size of the soft tissue structures that surround the upper airway in healthy younger $(\mathrm{n}=14)$ and older $(\mathrm{n}=11)$ males as measured from magnetic resonance images. a) Soft palate cross-sectional area, b) parapharyngeal fat pad diameter, c) parapharyngeal fat pad cross-sectional area and d) tongue cross-sectional area. Median data are denoted by horizontal lines on each plot.

as a risk factor for reduced pharyngeal calibre and propensity for collapse [12]. In addition, CHI et al. [23] found that hyoid bone displacement in OSA patients was dependent on BMI and tongue volume, and likely occurs secondary to soft tissue displacement. Therefore, our finding of no significant hyoid displacement with age may be a reflection of the well-matched groups with no difference in tongue volume.

The difference in pharyngeal calibre was also independent of bony box and tongue volumes. Reduction in craniofacial size has been associated with reduced airway calibre and a greater propensity to collapse [23]. It would, therefore, seem reasonable that a larger craniofacial size would be responsible for the large pharyngeal calibre seen in older participants in this study. However, no such difference was observed, suggesting that soft tissue anatomical changes and/or functional compensation may be responsible for the greater pharyngeal calibre in older participants. This would have occurred if some tissues increase in volume with age, while others decrease with age. Alternatively, the nonsignificant tendency for older males to have a slightly larger bony box volume may have reached significance with a greater sample size.

Despite the novelty of this study, there are several limitations that need to be acknowledged. The study sample size was small and participants were studied during wakefulness only. The small sample size reflects

\section{TABLE 3 Craniofacial parameters measured using magnetic resonance imaging}

Younger males

Subjects $n$

Bony box volume $\mathrm{cm}^{3}$

Tongue volume $\mathrm{cm}^{3}$

Bony box/tongue volume ratio

Nasion to hyoid $\mathrm{cm}$

Sella to hyoid $\mathrm{cm}$

Supramentale to hyoid $\mathrm{cm}$
14

$1010.2(929.4-1191.4)$

$731.3(651.2-800.2)$

$0.70(0.66-0.73)$

$12.7(12.1-13.3)$

$4.7(4.3-4.9)$
$11.6(10.7-12.2)$
Older males

11

$1110.2(968.0-1305.7)$

$737(653.9-787.1)$

$0.60(0.59-0.69)$

$13.3(12.6-14.2)$

$11.4(10.9-12.0)$

$4.7(4.5-4.8)$

Data are presented as median (interquartile range), unless otherwise stated. There were no significant differences $(p=0.05$ ) between groups for any parameter. 

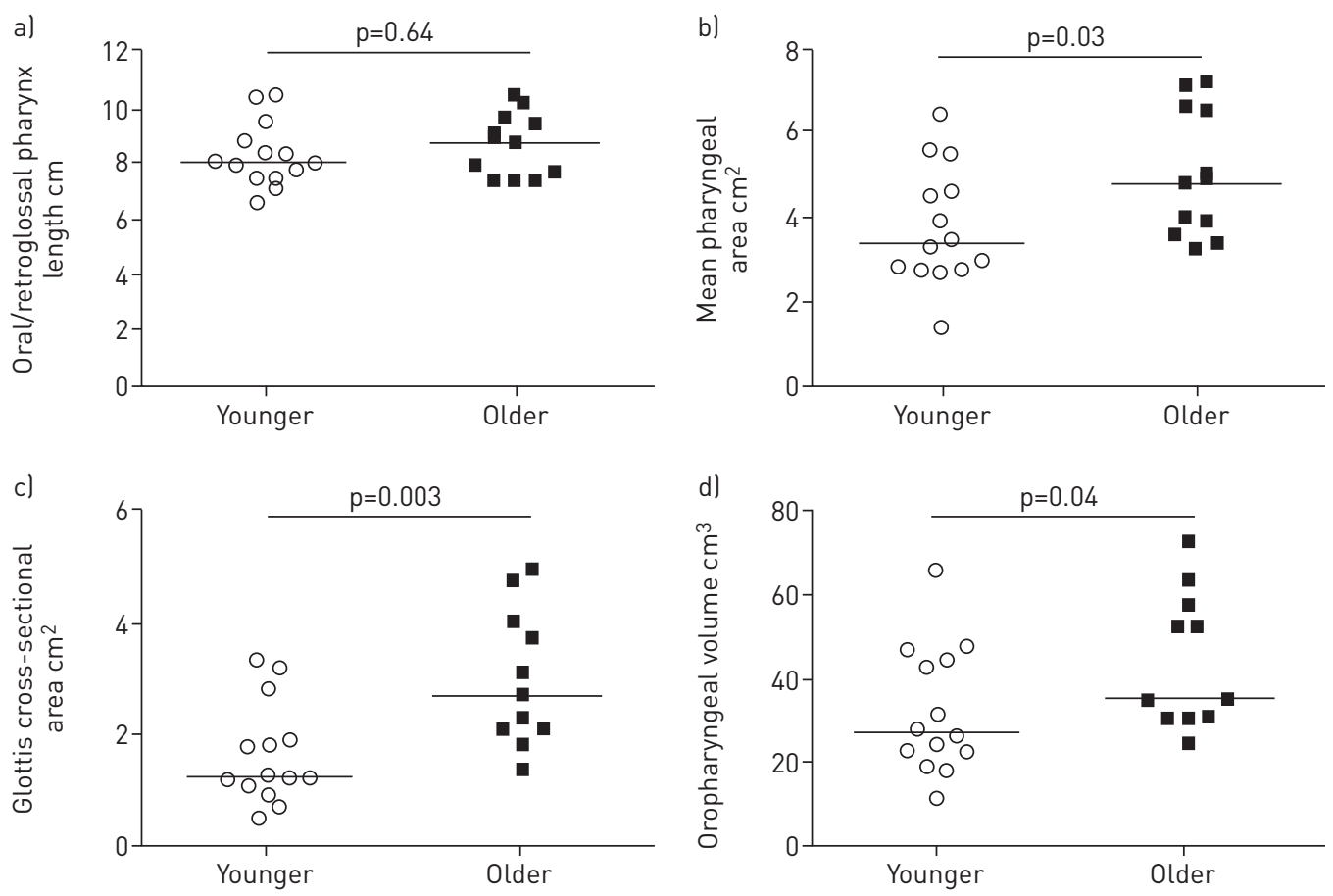

FIGURE 5 a) Oral/retroglossal pharyngeal length, b) mean pharyngeal area, c) glottis cross-sectional area and d) pharyngeal volume in healthy younger $(n=14)$ and older $(n=11)$ males as measured from acoustic reflection. Median data are denoted by horizontal lines on each plot.

the a priori decision to control for obesity by carefully matching our groups for neck circumference and BMI. This approach limited recruitment, although it also removed the need for retrospective statistical adjustments. A large effect size (Cohen's $\mathrm{d}=0.86$ ) was detected between groups for the mean pharyngeal area; likewise, the differences in combined retropalatal and retroglossal pharyngeal length, soft palate crosssectional area, fat pad diameter and fat pad cross-sectional area measured by MRI also had a large effect size (Cohen's $\mathrm{d}=1.08,1.00,0.87$ and 1.02, respectively). However, the retrospective power was $<0.80$ for all nonsignificant acoustic reflection and MRI parameters and, therefore, we cannot rule-out a Type II error. Logistical difficulties involved with sleeping in an MRI scanner, and maintaining consistent posture and an appropriate seal around the mouthpiece meant that studies during sleep were deemed beyond the scope of our resources. In addition, the known sex differences in fat distribution between males and females meant the decision was taken to study males only, allowing for careful matching of participants for both BMI and neck circumference.

Mouth breathing during both the acoustic reflection and MRI measurements limits the comparability of our findings to previous MRI studies with nasal breathing $[6,8,10,13,21,23]$, but allows direct comparison with previous acoustic reflection studies using mouth breathing [14, 16-18, 20, 24]. We reasoned that ensuring consistency in the arrangement of the pharyngeal structures between acoustic reflection and MRI measurements in our study would allow both modalities to complement one another, giving a more comprehensive overview of the upper airway anatomy. In addition, there was the potential for participants to breathe nasally during acoustic reflection and MRI measurements, resulting in different soft tissue arrangements between subjects. Participants were instructed to breathe through their mouth only. Acoustic reflection traces were excluded if nasal breathing was indicated by the pharyngeal cross-sectional area exceeding $15 \mathrm{~cm}^{2}$ (suggesting only partial elevation of the soft palate and escape of sound waves into the nasal passages). Mouth breathing during MRI was confirmed by observation of the soft palate abutting the posterior wall of the pharynx in all images.

This study provides insight into anatomical changes in the upper airway with age using two well-established methods. It provides a starting point for further research, including longitudinal studies for age-related changes in upper airway anatomy. Additional studies may include analysis of neuromuscular activity in the parapharyngeal musculature and the use of advanced imaging techniques such as magnetic resonance elastography to assess the biomechanical properties of the soft tissues implicated in this study. 


\section{Conclusions}

This study aimed to investigate the effects of age on pharyngeal morphology as a risk factor for OSA using healthy younger and older male participants matched for BMI and neck circumference. Older age was associated with greater combined retropalatal and retroglossal pharyngeal length, greater pharyngeal calibre, and larger soft palate and parapharyngeal fat pad size. We speculate that the unexpected finding of larger pharyngeal calibre may be a protective feature compensating for pharyngeal soft tissue encroachment that appears to occur in older people and predispose to OSA.

\section{References}

1 Peppard PE, Young T, Barnet JH, et al. Increased prevalence of sleep-disordered breathing in adults. Am J Epidemiol 2013; 177: 1006-1014.

2 Young T, Paltra M, Dempsey J, et al. The occurence of sleep-disordered breathing among middle-aged adults. N Engl J Med 1993; 328: 1230-1235.

3 Young T, Peppard PE, Gottlieb DJ. Epidemiology of obstructive sleep apnea: a population health perspective. Am J Respir Crit Care Med 2002; 165: 1217-1239.

4 Morrell MJ, Finn L, McMillan A, et al. The impact of ageing and sex on the association between sleepiness and sleep disordered breathing. Eur Respir J 2012; 40: 386-393.

5 Bradley TD, Brown IG, Grossman RF, et al. Pharyngeal size in snorers, nonsnorers, and patients with obstructive sleep apnea. N Engl J Med 1986; 315: 1327-1331.

6 Ciscar MA, Juan G, Martínez V, et al. Magnetic resonance imaging of the pharynx in OSA patients and healthy subjects. Eur Respir J 2001; 17: 79-86.

7 Dempsey JA, Skatrud JB, Jacques AJ, et al. Anatomic determinants of sleep-disordered breathing across the spectrum of clinical and nonclinical male subjects. Chest 2002; 122: 840-851.

8 Schwab RJ, Pasirstein M, Pierson R, et al. Identification of upper airway anatomic risk factors for obstructive sleep apnea with volumetric magnetic resonance imaging. Am J Respir Crit Care Med 2003; 168: 522-530.

9 Segal Y, Malhotra A, Pillar G. Upper airway length may be associated with the severity of obstructive sleep apnea syndrome. Sleep Breath 2008; 12: 311-316.

10 Malhotra A, Huang Y, Fogel R, et al. Aging influences on pharyngeal anatomy and physiology: the predisposition to pharyngeal collapse. Am J Med 2006; 119: 72.e9-72.e14.

11 Kollias I, Krogstad O. Adult craniocervical and pharyngeal changes - a longitudinal cephalometric study between 22 and 42 years of age. Part 1: morphological craniocervical and hyoid bone changes. Eur J Orthod 1999; 21: 333-344.

12 Pae E-K, Quas C, Quas J, et al. Can facial type be used to predict changes in hyoid bone position with age? A perspective based on longitudinal data. Am J Orthod Dentofacial Orthop 2008; 134: 792-797.

13 Horner RL, Shea SA, Mcivor J, et al. Pharyngeal size and shape during wakefulness and sleep in patients with obstructive sleep apnoea. Q J Med 1989; 72: 719-735.

14 Brooks LJ, Strohl KP. Size and mechanical properties of the pharynx in healthy men and women. Am Rev Respir Dis 1992; 146: 1394-1397.

15 Burger CD, Stanson AW, Sheedy PF, et al. Fast-computed tomography evaluation of age-related changes in upper airway structure and function in normal men. Am Rev Respir Dis 1992; 145: 846-852.

16 Mayer P, Pepin J, Bettega G, et al. Relationship between body mass index, age and upper airway measurements in snorers and sleep apnoea patients. Eur Respir J 1996; 9: 1801-1809.

17 Brown IG, Zamel N, Hoffstein V. Pharyngeal cross-sectional area in normal men and women. J Appl Physiol 1986; 61: 890-895.

18 Huang J, Shen H, Takahashi M, et al. Pharyngeal cross-sectional area and pharyngeal compliance in normal males and females. Respiration 1998; 65: 458-468.

19 Kollias I, Krogstad O. Adult craniofacial and pharyngeal changes - a longitudinal cephalometric study between 22 and 42 years of age. Part II: morphological uvulo-glossopharyngeal changes. Eur J Orthod 1999; 21: 345-355.

20 Martin S, Mathur R, Marshall I, et al. The effect of age, sex, obesity and posture on upper airway size. Eur Respir J 1997; 10: 2087-2090.

21 Malhotra A, Huang Y, Fogel RB, et al. The male predisposition to pharyngeal collapse. Am J Respir Crit Care Med 2002; 166: 1388-1395.

22 Iber C, Ancoli-Israel S, Chesson AL, et al. The AASM Manual for the Scoring of Sleep and Associated Events: Rules, Terminology and Technical Specifications. 1st Edn. Westchester, American Academy of Sleep Medicine, 2007.

23 Chi L, Comyn F-L, Mitra N, et al. Identification of craniofacial risk factors for obstructive sleep apnoea using threedimensional MRI. Eur Respir J 2011; 38: 348-358.

24 Rubinstein I, McClean PA, Boucher R, et al. Effect of mouthpiece, noseclips, and head position on airway area measured by acoustic reflections. J Appl Physiol 1987; 63: 1469-1474.

25 Hilberg O, Pedersen OF. Acoustic rhinometry: recommendations for technical specifications and standard operating procedures. Rhinol Suppl 2000; 16: 3-17.

26 Lo Y-L, Jordan AS, Malhotra A, et al. Influence of wakefulness on pharyngeal airway muscle activity. Thorax 2007; 62: 799-805.

27 Mezzanotte WS, Tangel DJ, White DP. Waking genioglossal electromyogram in sleep apnea patients versus normal controls (a neuromuscular compensatory mechanism). J Clin Invest 1992; 89: 1571-1579.

28 Worsnop C, Kay A, Kim Y, et al. Effect of age on sleep onset-related changes in respiratory pump and upper airway muscle function. J Appl Physiol 2000; 88: 1831-1839.

29 Fogel RB, Trinder J, Malhotra A, et al. Within-breath control of genioglossal muscle activation in humans: effect of sleep-wake state. J Physiol 2003; 550: 899-910.

30 Rama AN, Tekwani SH, Kushida CA. Sites of obstruction in obstructive sleep apnea. Chest 2002; 122: 1139-1147. 